\title{
TWO CASES ILLUSTRATING THE TREATMENT OF NEONATAL PNEUMONIA
}

BY

\author{
R. A. RATCLIFF, M.D.
}

A review of the pathological features of pneumonia in the new-born infant was recently published, and in that review Macgregor (1940) proposed a classification which would be equally useful clinically. The cases quoted below serve to illustrate the treatment of Macgregor's first type, namely that associated with aspiration of contents of the amniotic sac.

\section{Case reports}

Case 1. Baby K., a male infant, weighing $6 \mathrm{lb}$. $2 \mathrm{oz}$., was delivered after a long labour by an easy low forceps operation in the occipito-anterior position. The mother was a healthy primigravida and no abnormality occurred during pregnancy. At birth the infant was in a state of blue asphyxia, and an unusually large quantity of liquor amnii was drained from the mouth. Respiration became established without difficulty, but the lungs remained water-logged and air entry was restricted to the central and anterior parts of the lungs. Three hours after birth the infant breathed comfortably and there was no cyanosis, but respiration was noisy and there were râles to be heard over the anterior and upper parts of the lungs. At the bases and over the back there was little air entry and a few consonating râles.

For the first thirty-six hours of life the condition of the infant gave no cause for alarm. The colour was usually good, though there were attacks of cyanosis soon checked by administration of oxygen. No apparatus of the nature of an oxygen tent was available. During this time the infant was nursed on his right side to allow the left lung to become as free from fluid as possible, and the foot of the cot was raised. After the first eighteen to twenty-four hours no further liquor could be wiped from the mouth, and after twenty-four hours the motions passed contained a large proportion of vernix caseosa.

At the age of forty hours dyspnoea and cyanosis were much increased; oxygen was thereafter administered continuously through a nasal catheter. The moist sounds in the lungs were uniformly increased and the area of lung into which the air entered was much reduced. Alarming dyspnoea persisted for the next twelve hours and the distress then slowly diminished.

At the age of four days the left lung (lying uppermost) had appreciably greater air entry than before, though it was still very moist. The infant took small feeds of breast milk every two hours on this day, whereas only water had been taken until this time. No alteration of the baby's position was allowed.

At five days the infant breathed without effort and the moist sounds in the lungs were further reduced. Air entry into the right lung (lying downwards) became perceptible. On this day it appeared that the baby would probably recover if no infection developed in the still water-logged lungs. For this 
reason I prepared some serum from his father's blood and on the following day I gave 20 c.c. of this serum intramuscularly into the thigh.

Rapid improvement occurred during the next twelve hours, and at seven days old the baby was taking two-hourly feeds well and the oxygen was no longer required. The lungs had become very much drier since the injection and were expanded all over.

At eight days old no abnormality could be found, three-hourly breast feeds were instituted and no further precautions were taken.

The baby was discharged home on his eighteenth day weighing $6 \mathrm{lb} .10 \frac{1}{2} \mathrm{oz}$.

Case 2. Baby S., a male infant, weighing $7 \mathrm{lb} .14 \mathrm{oz}$., was born to a healthy multigravida after a short labour, delivery occurring immediately after rupture of the membranes without any delay at the vulva. A considerable quantity of liquor had been inhaled by the infant who was in a state of blue asphyxia. Mucus and liquor were drained from the mouth and, the baby being vigorous, respiration was satisfactorily established though the respiratory passages still contained fluid.

For the first fifteen hours the infant breathed well, and though there was a slight degree of cyanosis no attack of respiratory failure occurred. After this time air entry heard over the posterior surface of the chest was poor, and at the bases quite absent, both behind and in front ; air entry in the front and upper parts of both lungs was good, and neither general nor local conditions gave any cause for concern, because the lungs as a whole were well expanded even in the airless portions, and respiratory movements were powerful. The prognosis was then regarded as good. The foot of the cot was raised; oxygen was administered for ten minutes every hour and two-hourly feeds of breast milk were given by bottle.

No great change was seen until the infant was sixty hours old; there was no attack of cyanosis, the infant fed well and his motions were normal.

At sixty hours old the baby became peevish, dyspnoea and cyanosis increased, appetite failed and his motions contained insufficiently digested breast-milkcurds and mucus. There was only a slight increase of moist sounds in the chest, but it was clear that a super-imposed catarrhal infection had complicated the disorder, making the prognosis much worse. The infant was given 10 c.c. whole maternal blood (not citrated) into the thigh. Oxygen was directed to be continuously administered, feeds to be maintained as regularly as possible and the baby was to be nursed only on his right side. For the next twenty-four hours there was a slight improvement, but there was more mucus in the motions.

When the infant was ninety-six hours old alarming respiratory failure occurred; 1 c.c. coramine was given, and he slowly revived. An hour later there was an even more alarming collapse; breathing stopped, the heart-beats fell to sixty per minute, and blood-stained mucus poured from the mouth while oxygen was given by nasal catheter. No further injection of stimulant was made, but breathing was established once more and the infant received an injection of 12 c.c. of his father's blood into the thigh. The father was bled further for the purpose of preparing serum for future use.

The baby's general condition did not improve during the next twelve hours though only one attack of cyanosis occurred, less alarming than the former.

At four-and-a-half days old the left (upper) lung had a moderately good air entry, but breath sounds were accompanied by many râles, and blood-stained mucus was still coughed from the bronchi. The bowels were more relaxed. An intra-peritoneal injection of 30 c.c. of father's serum was given on this day.

At five days, after a further twelve hours, the infant was more comfortable, dyspnoea was much less and the baby was able to take an ounce of breast milk from a bottle every two hours with some enthusiasm and without increasing 
his dyspnoea. At this time air entry into both lungs was fair, râles were very much reduced, and oxygen was discontinued as there was no cyanosis and the nasal tube prevented sleep. The infant was still nursed on his right side.

At five-and-a-half days old 18 c.c. of father's serum was injected into the thigh.

At six days old there was good air entry into both lungs save for a small zone of airless lung at each base, more extensive on the right. Râles were still to be heard all over both lungs but were of a non-consonating character. Respiration was rather noisy, but there was neither dyspnoea nor cyanosis and the baby fed well. As the right lung was now almost equal to the left the infant could be nursed on either side with the cot flat, but was still fed without taking him up.

At seven days old no abnormality was to be found in his lungs and, in particular, there was no collapse even at the extreme bases. He fed from the breast well and the motions were fewer and contained little mucus.

At eight days old the chest was still normal. The motions were normal and the baby weighed only $7 \mathrm{oz}$. less than his birth weight. No further treatment was necessary and he was discharged home on his sixteenth day weighing 7 lb. $10 \mathrm{oz}$.

\section{Comment}

Diagnosis. As these infants both recovered there is no certainty that they were cases of pneumonia, and in infants recovering so quickly no more than very small areas of pneumonia can have existed. The clinical diagnosis rests upon the sudden deterioration in their condition and the coincident increase in physical signs throughout the lungs, though some of this increase should be attributed to the oedema shown by Henderson (1931), and later investigators, to be a necessary consequence of asphyxia. However, if neonatal pneumonia is to be treated with reasonable prospect of success this must be done at an early stage, which is well represented by the two cases described.

Treatment. The general treatment is not debateable, and the only comment to be made is that oxygen would have been more efficiently given with some form of oxygen tent, had it been available. The nasal catheter in a newborn infant is a source of troublesome increase of mucus secreted from the pharynx, and I therefore prefer that it should be inserted at intervals only, if the baby's condition permits.

The position of the infant employed to promote postural drainage is described, but this can only be expected to drain the trachea and bronchi. Surface tension alone is sufficient to prevent fluid draining from the bronchioles and alveoli without assuming that the smallest subdivisions of the lung are consolidated by inflammatory exudate. In each case no appreciable quantity of the inhaled liquor drained out after eighteen to twenty-four hours, though its presence was demonstrated by the absence of resonance and the character of the râles in a fully expanded area of lung.

Both these infants had reasonably well-expanded lungs, and this fact serves to confirm the statement made by Blaikley and Gibberd (1935) that intratracheal insufflation is of no use in treatment of neonatal asphyxia due to aspiration of large volumes of liquor amnii. If there had been any degree of atelectasis it would have been wise to attempt to promote expansion of the lungs very soon after birth to relieve embarrassment of the heart. 
The rapid improvement of each baby after injection of human serum was interesting. In each case the reason for this treatment was infection, threatened in the first and established in the second, but there is no evidence to show what was, in fact, the mode of action of this serum. The improvement took place so soon after the injection of a large dose that some immediate reaction in the infant's lung was probably responsible.

Macgregor (1940) in her account of the pathology of neonatal pneumonia refers to the oedema associated with the condition, and this oedema is much greater than in any comparable inflammatory process in the adult lung; the difference must surely be due to greater capillary permeability in the newborn infant. It follows that the simplest explanation of any such rapid improvement as that observed in these babies is the absorption of oedema fluid, without supposing that any true pneumonic process is suddenly reversed. I therefore believe that the injection of a large dose of serum diminished the capillary permeability and so reduced the pulmonary oedema resulting from asphyxia, whereas the later effect of increasing resistance to infection produced a less striking change.

There is no direct evidence which supports this view and I can only quote, in analogy, the rapid disappearance of some types of oedema neonatorum after injection of adequate doses of serum. If some experimental or other evidence should be obtained in support of this hypothesis, the conclusion would be that every newborn baby with any degree of dyspnoea associated with increasing moist sounds in the lungs should be treated by the injection of human serum in fairly large doses.

\section{Summary}

Two cases of neonatal pneumonia, associated with aspiration of contents of the amniotic sac, and ending in recovery, are described. The treatment is discussed with particular reference to the rapid improvement which followed injection of human serum.

\section{REFERENCES}

Blaikley, J. B., and Gibterd, G. F. (1935). Lancet, 1, 736.

Henderson, Y. (1931). J. Amer. med. Ass., 96, 495.

Macgregor, A. R. (1940). Arch. Dis. Childh., 14, 323. 